\title{
Mein Leben als Infarktpatient
}

Peter Marko

Korrespondenz:

Dr. med. Peter Marko Bruggwaldstrasse 39e CH-9009 St. Gallen

p.marko[at]bluewin.ch
Schon seit mehreren Monaten fehlte mir die Lust, schneller zu gehen, mich zu beeilen. Ich hatte keine Schmerzen, keine Atemnot, einfach keine Lust. Ich tröstete mich damit, dass ich, wie jeder, langsam älter werde und damit fertig werden müsse.

Eines föhnigen Sonntags Anfang September fuhren wir in Zweisimmen, wo ich zehn Jahre praktizierte und jeweils unter Föhn litt, mit der Gondelbahn auf den 2000 m hohen Rinderberg. Nach zwei-, dreihundert Metern bekam ich auf der linken Seite der Brust Druck, ähnlich wie vor Jahren bei Föhn, nur viel stärker. Ich dachte, der Föhn ist inzwischen, anders als ich, mächtiger und ich mit den Jahren empfindlicher geworden. Oben angekommen, kehrte ich mit der Bahn lieber sofort zurück. Und in der Tat, der Druck liess nach, aber verschwand nicht ganz. So weit weg von zu Hause wollte ich nicht ins Spital gehen. Zu Hause mass ich den Blutdruck - er war 160 systolisch und 95 diastolisch. Der Druck auf der Brust liess nach, und so beschloss ich, nichts zu überstürzen und bis zum nächsten Tag abzuwarten. Am nächsten Tag war ich beschwerdefrei, schleppte problemlos Lasten, trotzdem liess ich beim befreundeten Kollegen ein EKG aufzeichnen, das tadellos aussah. Mit dem Blut gab es unerwartete Probleme, sodass ich die Werte erst vier Tage später im Spital erfuhr. Nachts fuhr ich dorthin wegen diesmal kaum erträglicher Schmerzen.

Auf der Notfallstation war mein Blutdruck 230/113. Trotz immer noch nicht eindeutigen EKGZeichen, wurde ich schnell und ruhig behandelt, als ob ich einen Herzinfarkt hätte. Nach nicht ganz einer Stunde erhielten wir die neuen Laborergebnisse, die einen subakuten Infarkt zeigten, der den Werten nach offensichtlich bereits am Sonntag begonnen hatte. Um 8.00 Uhr wurde ich schmerzfrei auf die Abteilung verlegt. Es wurde mir angekündigt, dass mir mittags die verstopften Herzgefässe erweitert würden.

Auch nachdem ich von dem Infarkt erfahren hatte, blieb ich ruhig (schon vor der Morphiumspritze). Ich dachte, jetzt kann ich selbst nichts mehr für mich tun, Aufregung und Angst verschlimmern sowohl meinen Zustand wie die Arbeit meiner Betreuerinnen und Betreuer, denen ich völlig vertraute. Meine Ruhe und auch, dass ich keine Angst vor dem Tod spürte, überraschten mich. Vor mehr als dreissig Jahren, als ich wegen Verdacht auf Hodentumor operiert wurde, war ich nicht nur viel ängstlicher, sondern machte mir auch grosse Sorgen, was mit meiner jungen Familie geschehen würde. Jetzt war ich bereit zu sterben, da ich das Wichtigste in meinem Leben bereits erledigt und viel erlebt hatte, auch wenn ich nichts dagegen hätte, das Leben noch eine Weile zu geniessen, die Enkelkinder wachsen zu sehen. Merkwürdig -, die «Sünden», Versäumnisse, Fehler, Ungerechtigkeiten, Verstösse gegen Anstand und Moral, die ich sicher genug begangen hatte, kamen mir nicht in den Sinn, auch wenn ich früher dachte, sie würden mich in einer solchen oder ähnlichen Situation, so wie manchmal nachts, sicher plagen. Aber vielleicht war es noch nicht bedrohlich genug, und bei nächster Gelegenheit kommen sie doch.

\section{Das Wichtigste in meinem Leben hatte ich bereits erledigt.}

Auf der Bettenstation wurde ich von der Schwester - Entschuldigung - Pflegefachfrau liebevoll empfangen. Sie schlug mir aber gleich vor, dass sie mir beim Duschen helfe, was ich mit all den Schläuchen, Elektroden und Verbänden, dazu der Müdigkeit nach der halben Nacht ohne Schlaf, mit Schmerzen und Morphium dagegen, vielleicht spritzten sie mir auch Valium, nicht passend fand und entschieden ablehnte. Ich konnte dem wirklich keinen Sinn abgewinnen. Als sie insistierte, vermutlich wegen der Manipulation in der Leiste bei der Herzkathetrisierung mittags, was ich erst später begriff, verkündete ich, dass ich ein bekannter Schmutzfink sei, was mir leicht über die Lippen kam, da wir ein Closomat haben.

Vor der Kathetrisierung mit Angiographie wurde mir ans Herz gelegt, wegen der Kontrastmittelausscheidung genug zu trinken, was ich pflichtbewusst erfüllte. Als ich dann im Behandlungsraum wartete, weil der «Dilatator» zu einem Notfall gehen musste, füllte sich meine nicht mehr sehr elastische Blase immer mehr. Der Druck nahm bei der Desinfektion mit einer raumtemperaturkalten Flüssigkeit noch zu. Inmitten dieser sterilen «Hightechgeräte», wagte ich auf der Liege nicht, um eine Urinflasche zu bitten. So achtete ich nicht darauf, was mit meinem kranken Herz geschah, da ich mit dem Zustand meiner Blase und ihres Schliessmuskels beschäftigt war. Deswegen, oder eher wegen des Könnens des Kardiologen, spürte ich bei der Kathetrisierung und der Gefässerweiterung, auch wenn er immer wieder nachfragte, keine Schmerzen.

Nachmittags kamen die Enkelkinder zu Besuch. Die noch nicht dreijährige Enkelin ahmte die Kran- 


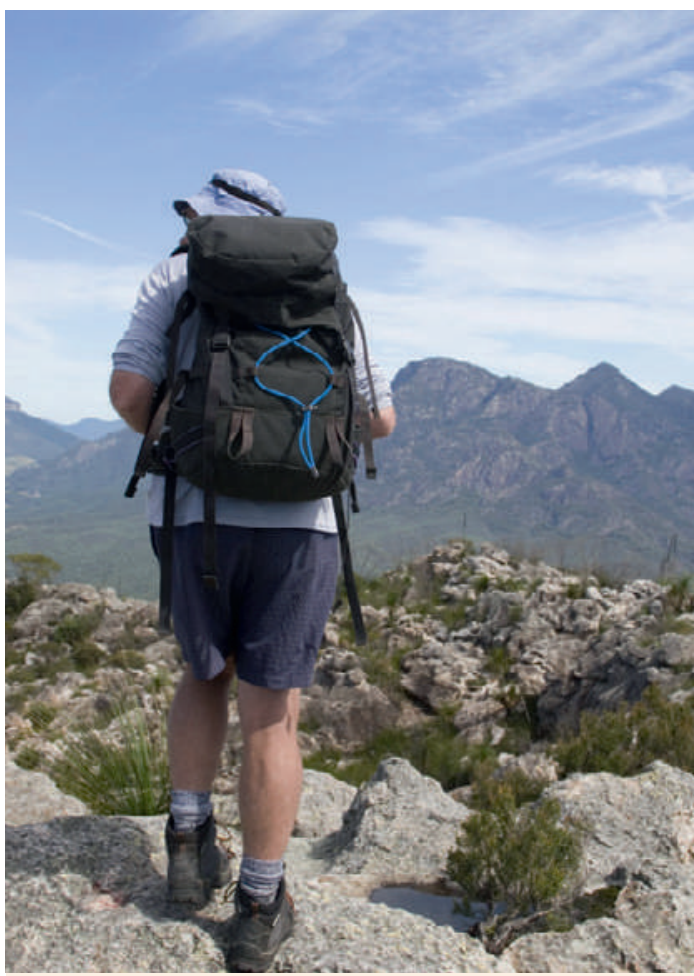

Jetzt geht es wieder: Bergwandern ohne Herzschmerzen.

kenschwester nach und forderte mich bei den kleinsten Bewegungen bestimmt auf, ich solle ruhig liegen bleiben, damit der Druckverband sich nicht verschiebe, und die Wunde nicht zu bluten beginne. Während der nächsten Nächte und auch am Sonntagmorgen spürte ich wieder ein ähnliches Ziehen wie im Simmental. Die Dienstärztin verordnete den Troponintest. Um das Blut dazu abzunehmen, kam eine Schwester in Ausbildung. Offensichtlich waren die erfahrenen Pflegerinnen am Sonntagmorgen mit anderen Aufgaben voll beschäftigt. Meine nicht gerade bäumigen Venen waren schon durch die vielen Stiche und Blutungen kaum auffindbar, und ich zweifelte, ob eine noch nicht sehr erfahrene Pflegerin Erfolg haben würde, aber behielt es für mich. Nach drei zusätzlichen Stichen, die von mir heroisch ohne Zuckungen ertragen wurden, sagte sie, sie müsse den Pfleger rufen. Nach zwei, drei vergeblichen Versuchen wollte er es aufgeben. Ich riet ihm, es an einer meiner Beinkrampfadern zu versuchen. Offensichtlich kannte er diese Quelle noch nicht. Ich stand auf, er legte den Stauschlauch an, und so bekam er genug Blut. Der Test war negativ. Als ich wegen des Ziehens in der nächsten Nacht erwachte, belastete ich damit niemanden mehr. Schon am vierten Tag wurde ich mit vier Medikamenten, zwei davon gegen hohen Blutdruck, nach Hause entlassen. Mit der Betreuung im Spital war ich äusserst zufrieden.

Nachdem sich das Ziehen zu Hause jede Nacht wiederholt hatte, mass ich den Blutdruck und stellte fest, dass er jeweils dabei um 160 systolisch war, we- sentlich höher als tagsüber. Da ich ein eifriger, leidenschaftlicher Schnarcher bin, dachte ich zuerst an Schlafapnoe als Grund dafür, aber während der ambulanten Somnografie schnarchte ich zwar häufig und kräftig, aber ohne eine einzige Apnoe. Zuerst nahm ich alle blutdrucksenkenden Medikamente am Abend. Dann begann ich, mit den Medikamenten und ihrer Dosierung zu experimentieren. Mit Erfolg. Ich nehme jetzt vier Antihypertensiva, dazu andere Medikamente, insgesamt viermal täglich. Als ich versuchte, diese zeitliche Einteilung zu vereinfachen, stieg wie die Ahle aus dem Sack der Blutdruck irgendwann in den unerwünschten Bereich auf.

So ein kompliziertes Regime hätte ich einem Patienten kaum zugemutet. In der Hoffnung, dass sie sich dadurch eher daran halten, zwang ich Patienten mit ihren verschiedenen Stoffwechseln, Hormonhaushalten, psychischen und körperlichen Rhythmen, Darmbakterien in ein möglichst einfaches Schema, anstatt das Schema ihrem Körper anzupassen. Ein alter, nicht mehr tätiger Arzt kann über sein Verhalten, seinen Arbeitsstil, seine Tätigkeit nur sinnieren, er kann nichts mehr ändern, verbessern. Es gibt darüber einen wunderbaren, rührenden Film Bergmans «Wilde Erdbeeren», der dazu beitrug, dass ich einen grossen Teil meines Lebens als Allgemeinarzt verbrachte.

Bereits im Spital wurde mir mit Nachdruck nahegelegt, dass ich mich einer ambulanten kardialen Rehabilitation unterziehen sollte. Ich war nicht begeistert, da ich dachte, ich turne (fast) jeden Morgen, laufe auch bergauf (fast) jeden Tag, und sich von Maschinen in einem geschlossenem Raum foltern zu lassen, entspricht nicht meinen «Naturneigungen». Aber widerspenstig und undankbar wollte ich mich nicht zeigen, und so begann ich, zweimal pro Woche, wie Don Quichotte mit den Windmühlen, meinen Kampf mit den Fitnessmaschinen, die wie Gespenster lauerten. Während Don Quichotte dabei übel zugerichtet wurde, wurde ich immer stärker und konnte morgens besser und länger turnen. Einmal pro Woche, am Mittwoch, sind wir in der Natur anderthalb Stunden ziemlich zügig marschiert.

Jetzt laufe ich die Hänge hinauf durch die Wälder so schnell wie schon lange nicht mehr. Ich hoffe, es bleibt eine Weile noch so, und bin allen dankbar, die dazu beigetragen haben. Ich schätze es sehr, dass die nicht geringe finanzielle Last der ganzen Behandlung von der Allgemeinheit fast vollständig übernommen wird. Während der Krankheit wunderten sich nicht wenige, wie es möglich ist, dass ich mit einer fast zwanghaft gesunden Lebensweise einen Herzinfarkt erleiden konnte. Mehrere Teilnehmer in der Rehabilitationsgruppe sagten, ihnen sei es gleich ergangen. Aber so kann ich mit einem besseren Gewissen die Kostenträger belasten, und allem Anschein nach verstopfte sich das Gefäss dadurch später, der Infarkt war kleiner und der Verlauf günstiger. 